\title{
Medium Access Control in Large Scale Clusters for Wireless Sensor Networks
}

\author{
P. Barbosa N. M. White N. R. Harris \\ Electronic Systems and Devices Group \\ School of Electronics \& Computer Science \\ University of Southampton, UK
}

\begin{abstract}
One of the main advantages of clustering algorithms is the ability to allow networks to be physically divided into smaller regions, thereby increasing the theoretical maximum number of nodes connected in the network. However, the physical limitations in bandwidth and number of nodes are drawbacks to this approach. While reducing the number of clusters can reduce the network complexity, it will increase the intra-cluster management.

This paper analyses the different medium access schemes that can be used and discusses what influence they bring to the overall performance in terms of bandwidth usage, energy consumption and latency. Two novel approaches are also proposed. The first one refers to the limitation in the transmission period and the request for each node within the cluster to transmit once within that period. The second innovation compromises two of the access mechanisms described: the first divides the period in two different parts, while the second takes different bias during the slot selection process, so it can have theoretical advantage over the re-transmission procedure. The results show that there is a significant advantages in listening to the channel prior to the transmission, as well as in re-trying to transmit only once.
\end{abstract}

\section{INTRODUCTION}

Localised maritime monitoring described in [1] describes a cluster network to be deployed on the water, in the case of a chemical spill. It is a technique that aims at distributing energy usage more evenly across the network by using simple and efficient routing protocols with low latency. The main focus went to the intra-cluster communication, as it provides the basic starting point for a large network. Moreover, by being logically - and sometimes even physically - independent from the upper routing levels, each cluster tier can be considered separate in its architecture.

Clustering protocols require that Cluster Heads (CHs) use different schedules from the rest of the nodes, as they must to send all the received information across the network to the sink node. To allow this, the $\mathrm{CH}$ must either be equipped with two different transceivers or divide its communication time between intra and inter cluster communication. Moreover, in the case of sensors deployed at sea, as the sea conditions can change dramatically, the connectivity can be very low at times. Therefore, if the $\mathrm{CH}$ has means to predict how the connectivity can be affected by sea surface condition, it may also use that information to advertise the transmission period for nodes to send their messages.

Medium Access Control (MAC) algorithms are an essential part of any network stack. They make it possible for different nodes to access the communication channel, providing mechanisms to detect and avoid other communications. By analysing the performance of different MAC algorithms, conclusions can be drawn about each one's performance. The differences will reflect not only in the number of delivered messages, but also in the bandwidth usage, energy needed to deliver those messages and the number of collisions (partially due to hidden node problems). Ultimately, there is a trade-off between the energy per bit needed to deliver each message and the number of messages that are delivered correctly.

This paper provides a study on the behaviour of large scale clusters and discusses the best alternatives for nodes to transmit the sensed information across the network to a sink node. It addresses the probability of collision in the cluster and, more specifically, each transmission. Three different aspects of communication are considered: contention, re-transmission and hidden node problem. The comparison between them will be provided in terms of successful delivery rate and energy usage. In addition, the limitations to the connectivity period confine the amount of time that nodes have to communicate with the $\mathrm{CH}$. Hence it is fundamental to understand the behaviour of the network under low connectivity deployment.

\section{A. Related work}

One of the first proposed MAC algorithms is Sensor-MAC (S-MAC), described by Ye et al. [2], [3]. It is essentially a Request-To-Send/Clear-To-Send (RTS/CTS) scheme where nodes share a common communication and sleep schedule in a virtual cluster. Using RTS/CTS also improves message delivery by reducing the collision probability. Nevertheless, RTS/CTS scheme has drawbacks: it cannot be used for broadcasting, energy consumption is greater than that of other approaches due to overhearing and idle listening, and it demands greater overhead.

WiseMAC was proposed as part of the WiseNET framework [4]. It uses a non-persistent Carrier Sense Multiple Access (CSMA) with a preamble preceding the message. The nodes have a periodic sleep/wake-up schedule, yet there is no guarantee that the receiver will be ready to receive the packet by the end of the preamble, causing over-emission energy waste. Clock drift is predicted and dealt with by using adaptive preamble. Moreover, there is no method of avoiding the hidden node problem.

The Traffic-Adaptive Medium Access protocol (TRAMA) uses Time Division Multiple Access (TDMA) for energy 
efficiency [5]. The slot distribution is performed for all nodes within a two-hop range. There are three potential types of access: predicted, random and scheduled. By using TDMA the contention and collision by hidden terminal problems are significantly reduced, resulting in improved energy savings. However, the transmission slots defined are seven times larger than the random access period, and the delays are higher than those in contention-based protocols.

DMAC departs from the observation that networks frequently use unidirectional paths to send the information to sinks so that they create an improved version of slotted ALOHA algorithm [6]. Its main objective is to reduce latency by using a data gathering tree that assigns subsequent slots to nodes in successive data transmission paths. The biggest drawbacks come from not using any collision avoidance method, and the overhead and complexity to create and manage data gathering trees.

Methley et al. [7] discuss some of the myths about mesh networking. The authors state that meshes do not work as expected in the literature, and even the addition of a new node does not bring additional capacity to the network. In the worst case scenario, it may not even become part of the network. The real advantages of using mesh networks come from the extended coverage and the ability to hop messages when obstacles compromise connectivity. Their conclusions refer to flat networks and do not fully extend to the routing protocols found in WSNs.

Node distribution in simulations is commonly assumed to be completely uniform; sometimes with nodes placed in a square grid and with uniform traffic [8], [9]. Real deployments require nodes placed in strategic positions (in industrial environments, for example) or by randomly deploying them on a region. In both these cases, the displacement will not be completely uniform and the load sharing will not be evenly distributed.

All existing MAC algorithms predict that there is no unexpected interruption in the medium access, unless for multiplexing purposes. That might not be the case when using clustering algorithms, such as in the application scenario described. In clustering, that assumption is only possible if the $\mathrm{CH}$ possesses two or more transceivers for intra and intercluster communication, otherwise it will have to schedule different time periods for each of them. On the other hand, the connectivity periods are limited, and if the $\mathrm{CH}$ has means of predicting the channel availability, it can make the best use of this function so that all the nodes benefit from it. Taking this into account, this report compares different access mechanisms when such conditions occur.

\section{LARGE SCALE CLUSTERS}

Scalability can be described as a property of a system that indicates the change in the throughput in relation to the change in system resources. Being more specific, scalability refers to the capacity of a network to manage variation in the number of nodes, while keeping the total throughput proportional to that variation. The network performance and scalability constraints will be affected by application-specific requirements, as well as the deployment environment.
One of the central issues with large scale clustering is the ratio between $\mathrm{CHs}$ and sensing nodes. Increasing the number of clusters provides more distributed management, at the cost of increasing complexity in upper tiers. Larger clusters, on the other hand, reduce the network management and overhead in upper tiers, at the cost of increasing contention inside each cluster [10], [11], [12].

If the number of nodes and covered area of a WSN increases, the management complexity also increases. Conversely, the end-to-end performance is gradually affected, down to the point where the network becomes unusable [13]. Routing protocols are seen as the first alternative to improve scalability of the network, since they provide tools for the nodes to decide which neighbours will provide the best service. As such, the following considerations were made for this research:

- Transceivers with improved Signal-to-Noise Ratio (SNR), such as the RFM LPR2400 [14] can increase transmission range, resulting in wider clusters, when compared to standard 802.15.4 transceivers.

- Large cluster size reduces the total number of clusters in the network at the cost of more nodes contending for the same $\mathrm{CH}$.

- Contention-based channel access provides improved energy and bandwidth usage when compared to RTS/CTS access. Virtual time slots (such as slotted ALOHA) and collision avoidance schemes will be used to increase robustness.

\section{A. Channel access in large scale clusters}

When a network uses a contention based protocol, only the nodes within transmission range will avoid communicating at the same time. All the others can transmit their data, resulting in hidden node collisions. If, on the other hand, a RTS/CTS scheme is used, the hidden node problem is eliminated at the expense of increased overhead and exposed terminal problems. As the transmission period is limited, there are important aspects to consider when selecting the channel access mechanism [15], [2], such as energy, re-transmission and back-off mechanisms, redundancy, overhead and collisions. A trade-off between them represents a balance between communication reliability, bandwidth and energy usage to better suit each application requirements. In large scale networks, minimising communication overhead has added importance compared to the other aspects. For that reason, simple protocols are more relevant, as long as they can guarantee high data delivery success.

\section{COLLISIONS AND HIDDEN NODES}

In a large clustered network, nodes are logically assigned to a given $\mathrm{CH}$ and will transmit the sensed data to it. A challenge in large scale WSNs is packet collision, resulting from two or more nodes sending data at the same time to destinations in-between. MAC protocols have been developed to minimize collisions by avoiding them through strict schedules or through contention access. To support the collision avoidance decision 
IEEE 802.14.5 [16] provides both scheduled and contentionbased protocols.

Schedule and contention-based protocols present different limitations and trade-offs. A scheduled protocol attributes a different time slot to each node, letting nodes to switch off their transceivers while waiting for their assigned slot, reducing energy waste and eliminating collisions. Yet, scheduling requires frequent updates of connected nodes, new node search, slot re-distribution, and synchronisation packets. This reflects in increased overhead and energy distribution. In addition, the number of slots is limited and pre-allocated, resulting in limited number of nodes that can belong to the cluster.

By listening to the channel before transmitting, contentionbased protocols try to minimise collisions between nodes. This procedure also helps reducing re-transmissions and saving energy. Channel allocation is not compulsory, which also reduces communication overhead and network setup requirements. Instead, the schedule is completely distributed and the nodes rely on each one acting differently from the others when choosing a transmission time. Given their simple nature, contention-based algorithms will be further investigated.

\section{A. Collision estimation}

Consider a network of $N$ nodes where $N=\left\{n_{1}, n_{2}, \ldots, n_{k}\right\}$ and $k$ is a number between 1 and 1000. Each node requires $t_{T x}=10 \mathrm{~ms}$ to send each message (corresponding to less than half the transmission period if transmitting at 250kbps). The period of time $T$ available for each node to transmit a message is between 30 and 300 seconds (a value based on a calm to moderate sea conditions on high sea), and it is repeated indefinitely. The maximum number of time slots achievable in these conditions is 3,000 and 30,000 , for $T=30 \mathrm{~s}$ and $T=300 \mathrm{~s}$, respectively. To avoid every node transmitting at the same time, each node will select its own transmission slot based on a random number. Using this information, it is possible to predict the probability $p(n)$ of any two nodes sending messages at the same time, based on the birthday problem [17]. A simplified version of the formula is

$$
p(n)=1-e^{-\frac{t(t-1)}{2 T}}
$$

where $t$ is the maximum number of consecutive messages that can be sent without collision, i.e. $t=T / t_{T x}$. The probabilities for $T=30 \mathrm{~s}, T=120 \mathrm{~s}$ and $T=300 \mathrm{~s}$ are as shown in figure 1.

Equation 1 shows the probability of collision occurrence between at least two nodes at any time. Yet, it does not specify which nodes will be colliding. For a given node $n_{i}$, equation 2 shows the probability $q\left(n_{i}\right)$ of any other node in the network transmitting at the same time.

$$
q\left(n_{i}\right)=1-\left(\frac{T-1}{T}\right)^{N} .
$$

The resulting graph is shown is figure 2. The parameters used were the same as in figure 1. It becomes clear that by using a large $T$ the probability of message collision for each

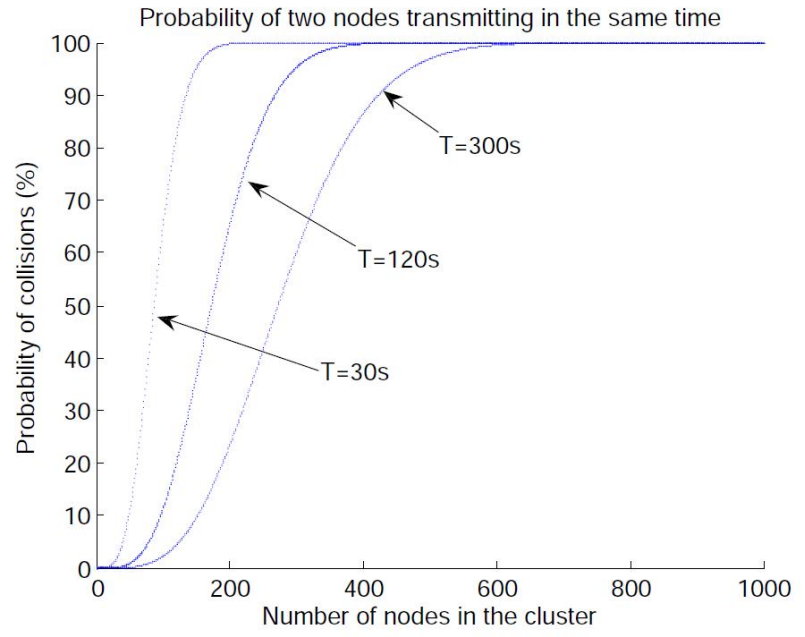

Fig. 1: Probability of collision for any two nodes in the network

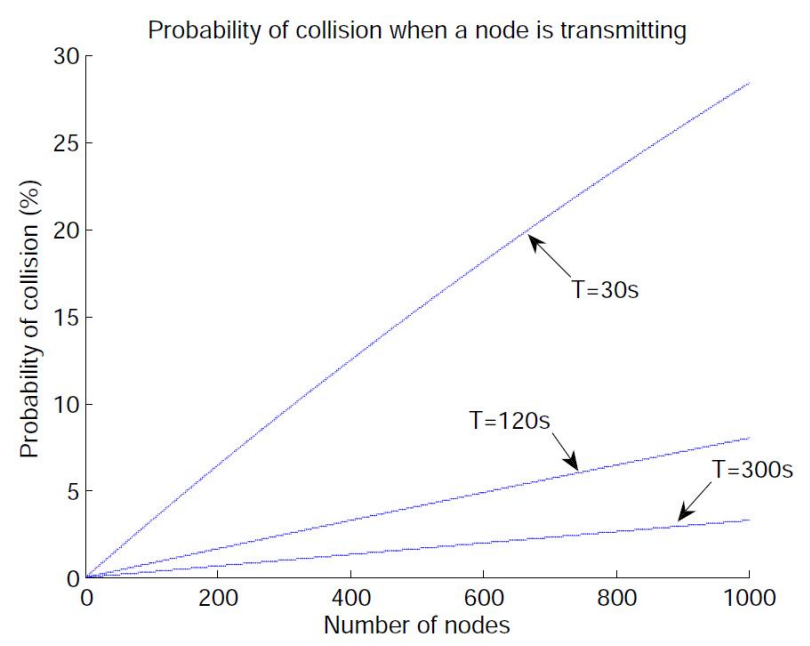

Fig. 2: Probability of collision when a given node is transmitting

node is relatively small. However, in the case where nodes can avoid collisions by listening to the channel before transmitting, the probability of collision $q_{r}\left(n_{i}\right)$ is reduced by the coverage of the nodes, resulting in

$$
q_{r}\left(n_{i}\right)=q\left(n_{i}\right) \cdot \operatorname{cov},
$$

where cov is the percentage of nodes within reach.

It becomes clear that the transmission period must increase exponentially with the number of nodes to keep the same probability of collisions across the network. On the other hand, as $q\left(n_{i}\right)$ increases in a relatively linear manner, the period may also increase linearly with the number of nodes to keep the collision constant for any given node.

It can be said that if a node $n_{i}$ transmits successfully at time slot $t_{j}$, it's because it didn't succeed in the previous $t_{j}-1$ time slots. Using a geometric distribution, it is possible to estimate how many time slots are needed before node $n$ transmits 
successfully. If $p$ is the probability of a node transmitting in a given time slot, then $1-p$ is the probability of not transmitting in that time slot. As such, for a given time slot $t_{j},(1-p)^{t_{j}-1}$ is the probability of $n_{i}$ not transmitting in the previous $t_{j}-1$ time slots. Hence, equation 4 gives the probability of each node $n_{i}$ transmitting in $t_{j}$.

$$
p\left(t_{j}\right)=p \cdot(1-p)^{\left(t_{j}-1\right)}
$$

As part of a network with $N$ nodes, $p\left(t_{j}\right)$ should not be considered a unique event. Indeed, the complete network transmission average for each time slot can be calculated as

$$
p\left(N, t_{j}\right)=N \cdot p \cdot(1-p)^{\left(t_{j}-1\right)} .
$$

\section{Medium access StRategies}

When a node wants to transmit its data, it may or may not listen to the channel before for any transmission. If it does so, it may reduce the probability of collisions. However, if the number of collisions is small, the gain might not be relevant enough to justify the extra energy used. To analyse this, a comparison between the different medium access mechanisms is made. Four basic methods of medium access will be used:

1) Simple access without channel listening. This method leads to both the maximum number of collisions and simple energy saving (one message sent every time period, without any channel listening). This is the basic metric for collision estimation under the conditions described above.

2) Basic contention-based method. Using a slotted access, each node listens to the channel before transmitting. If the channel is being used, the node does not transmit anything during that period of time. The purpose is to have an idea of the basic energy saving mechanism, while avoiding collisions with other nodes within range.

3) Simple re-transmission mechanism. The transmission period is divided in two parts. The nodes try to transmit sometime in the first part of the period and, if any potential collision is detected while listening to the channel, they will choose another time during the second part. This is a very simple mechanism for collision avoidance and re-transmission that should provide indication of the improvements achievable by using a backoff mechanism.

4) Multiple re-transmissions. When nodes detect a potential collision, they select another time slot until the end of the period. Three different curves will be used to distribute the slot selection probability. This method increases the number of tentatives allowed to each node at the cost of extra energy consumption.

The simple re-transmission mechanism divides the period $T$ in two main sections, $T_{1}$ and $T_{2}$, as can be seen in figure 3 . All nodes try to communicate by choosing a time slot from $T_{1}$ and, if another transmission is detected in the same slot before transmitting, the node will choose another time slot from $T_{2}$. The distribution of time between $T_{1}$ and $T_{2}$ can be modified to achieve the best result.

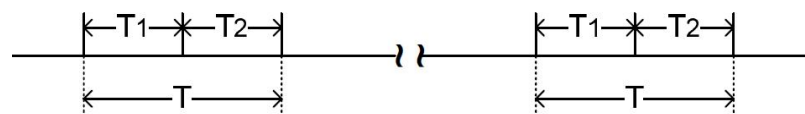

Fig. 3: Simple re-transmission mechanism based on the division of $T$ into two periods

For this case, the expected probability of success relies on the success of each time period independently. If $p\left(T_{1}\right)$ and $p\left(T_{2}\right)$ are the probabilities of collision avoidance for each period, then $p\left(T_{2}\right)$ will depend on the transmission success and collision rates of $p\left(T_{1}\right)$, as the nodes remaining to transmit during $T_{2}$ will be $N-\left(N \cdot p\left(T_{1}\right)\right)$. This is also dependent on the transmission range of each node and the amount of nodes they can reach from those that communicate with the same $\mathrm{CH}$.

The unlimited re-transmission process removes any previous restriction the number of transmissions in one period, so that each node can retry sending its data while there is time left. To assist with the time slot selection, a curve fitting model based on the idea show in figure 4 was attempted, with different curves being tried. In these models, each node chooses a time slot based on a probability $p$ for each. When the transmission period $T$ starts, the node has the possibility of choosing any time slot. If it decides to use slot $t_{1}$ and by some reason the transmission was unsuccessful, it will randomly choose another slot from that moment onwards. To distribute $p$ more evenly, when nodes have to select another slot from $T / 2$ onwards, the curve of $p$ is mirrored.

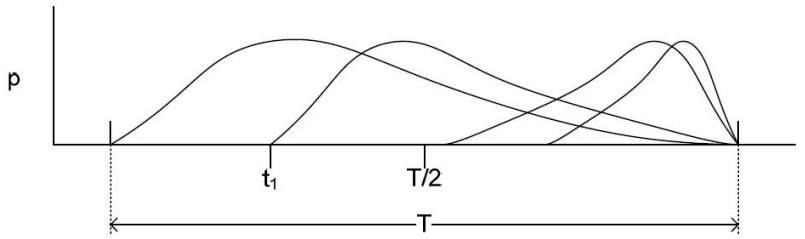

Fig. 4: Multiple re-transmission mechanism with skewed curves

Three different distributions were used for the curve fitting, representing different probabilities of slot selection:

- The first distribution is completely flat, therefore the nodes choose any time slot without any preference.

- The second distribution is highly skewed around $25 \%$ of the remaining time for the first half of the period, from where the curve becomes more skewed to $75 \%$ of the remaining time. A representation of the distribution can be seen in figure $5 \mathrm{a}$.

- The third distribution works in the same manner as the second, with the highest probability around $25 \%$ and with mirrored curve after $T / 2$. The main difference is that the curve is flatter, as can be seen in figure $5 \mathrm{~b}$.

The curves are skewed around $25 \%$ of the total period to reduce the number of nodes transmitting during the last slots, therefore reducing contention. On the other hand, mirroring 


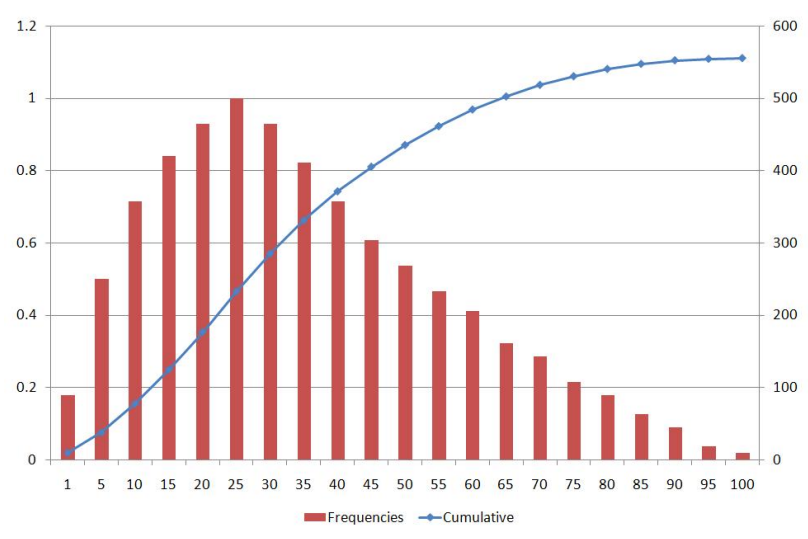

(a) Curve 1

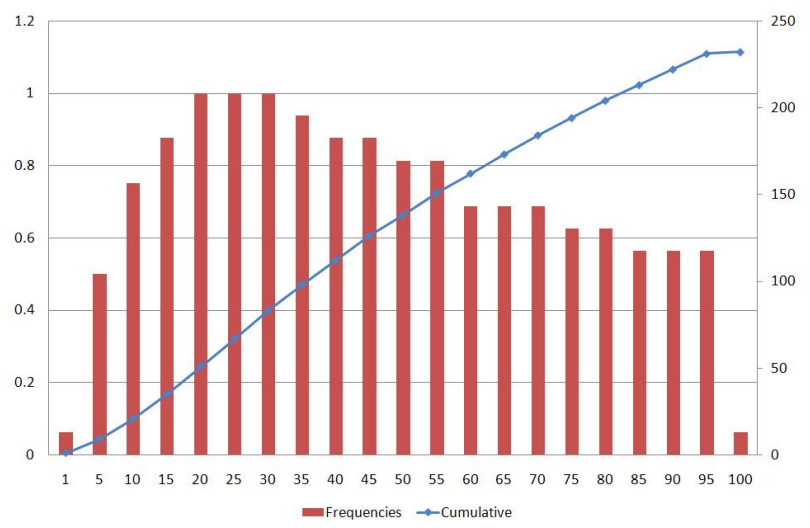

(b) Curve 2

Fig. 5: Two curves used for the probability distribution across the period

the distribution after $T / 2$ will ensure that the retransmissions are made towards the end of $T$, avoiding the more congested areas.

The algorithms were based in two different variations. The first is with nodes transmitting at maximum power, independently of their distance to the $\mathrm{CH}$. In the second, each node transmit with the minimum power for a message to correctly reach the $\mathrm{CH}$. Although in real deployment nodes always need to transmit above the minimum theoretical power, this is the extreme scenario where more nodes will be out of the contention range.

\section{Simulations AND Results}

For the implementation, a simple random number was used to select which time slot to use. Also, to define when two or more nodes transmitting in the same slot should go into contention or simply collide, the area of two intersecting circles was used. If $A_{C H}$ the transmission range of the $\mathrm{CH}$ and $A_{n}$ is the range of node $n_{i}$, then the average detection and avoidance of other nodes' transmissions is:

$$
p_{r}\left(t_{j}\right)=p \cdot(1-p)^{(t-1)} \cdot N \cdot\left(\frac{A_{C H} \cap A_{n}}{A_{C H}}\right)
$$

Having the intersection of two areas and considering nodes are uniformly distributed across the network, equation 2 shows that the probability of the remaining nodes trying to communicate at the same time as node $n_{i}$.

Simulations were created in Matlab where it was possible to compare how the different techniques behave. The physical layer was considered ideal and all nodes were within range of the $\mathrm{CH}$. As such, the different proposals represent the adaption of generic algorithms, such as slotted ALOHA (transmission without previous channel listening) and CSMA/CA (transmission with channel listening). The innovation resides in the unlimited re-transmission method using curve fitting models to distribute the load throughout the period, in an attempt to improve message delivery. The curves resemble a Poisson distribution of traffic, with the difference of being calculated according to the remaining transmission time.

To analyse the energy consumption, the values for each event were mostly based on the Chipcon CC2430 radio [18], and were as described in table I. For the sake of simplicity and general-purpose, the different transceiver currents will be considered to be the same.

TABLE I: Simulation parameters

\begin{tabular}{ccc} 
Parameter & Value & Units \\
\hline Number of nodes & 1000 & \\
Transmission period & 30 & $\mathrm{~s}$ \\
Message size & 1024 & bits \\
Transmission time & 10 & $\mathrm{~ms}$ \\
Listening time & 200 & $\mu s$ \\
Switching Tx/Rx time & 192 & $\mu s$ \\
$I_{T x}, I_{R x}, I_{s w}$ & 20 & $\mathrm{~mA}$ \\
\hline
\end{tabular}

To assess the performance of the algorithms, several different versions of MAC protocols were implemented, one without any contention and all the others with contention mechanisms. Furthermore, different rules for time slot selection and communication range were also implemented. All results were compared in terms of successful message delivery and average energy usage per delivered bit. The simulation was divided into two stages. During the first stage three different approaches were studied: transmission without previous listening, transmission with previous listening but no back-off mechanism, and the single re-transmission mode.

Table II shows the different results when each node tries to transmit only once. It is visible that listening to the channel improves message delivery, especially when using maximum transmission power. This is due to the number of nodes aware of the ongoing transmission being larger for higher power communication.

Listening to the channel before transmitting improves message delivery by $8 \%$. This comes from the nodes detecting potential collisions while listening to the channel and stopping from transmitting. This decision not only improves overall message delivery, but also helps saving energy. Using the values of table I, the total energy per period used by noncontention method was calculated to be approximately $200 \mathrm{~mJ}$ 
TABLE II: Comparison of messages delivered when using a single transmission, with and without listening

\begin{tabular}{ccc} 
Access method & Delivered & Colliding \\
\hline No contention & $71.5 \%$ & $28.5 \%$ \\
Contention, min $P_{T x}$ & $75.2 \%$ & $20.3 \%$ \\
Contention, $\max P_{T x}$ & $79.5 \%$ & $11.3 \%$ \\
\hline
\end{tabular}

for maximum transmission range and $155 \mathrm{~mJ}$ for the minimum transmission range, while the total energy used if listening to the channel is $152 \mathrm{~mJ}$ for the minimum transmission range and $185.5 \mathrm{~mJ}$ for the maximum transmission range.

Observing that nearly $80 \%$ of the nodes transmit successfully when listening to the channel to avoid collisions, the simple re-transmission algorithm periods were empirically divided as $T_{1}=80 \%$ of total time, leaving the remaining $20 \%$ for $T_{2}$. Table III shows the success rate of this method, using minimum and maximum $P_{T x}$. They show improvements of $6 \%$ for maximum $P_{T x}$ and $1.4 \%$ when $P_{T x}$ is the minimum.

TABLE III: Comparison of messages delivered when dividing the total time period in two stages

\begin{tabular}{ccc} 
Access method & Delivered & Colliding \\
\hline & & \\
Two transmissions, min $P_{T x}$ & $76.6 \%$ & $23.3 \%$ \\
Two transmissions, $\max P_{T x}$ & $85.6 \%$ & $13.6 \%$ \\
\hline
\end{tabular}

Table IV provides the results of the multiple re-transmission algorithms. It is visible that the flatter the curve is, the better the results are. This is due to the lower concentration of transmissions in one region, which reduces the probability of collisions. Moreover, the number of nodes retransmitting is smaller when using a flat distribution. The flat distribution is the only one achieving better delivery rate than the single retransmission scheme, by $0.4 \%$.

TABLE IV: Multiple re-transmission access methods, with percentages of overall successful nodes and percentage of nodes retransmitting

\begin{tabular}{ccc} 
Access method & Delivered & Retrying \\
\hline & & \\
Flat distribution, min $P_{T x}$ & $77.1 \%$ & $5.3 \%$ \\
Flat distribution, $\max P_{T x}$ & $86.0 \%$ & $12.1 \%$ \\
1st. distribution, $\min P_{T x}$ & $72.1 \%$ & $6.7 \%$ \\
1st. distribution, $\max P_{T x}$ & $82.5 \%$ & $14.7 \%$ \\
2nd. distribution, $\min P_{T x}$ & $75.4 \%$ & $5.5 \%$ \\
2nd. distribution, $\max P_{T x}$ & $85.1 \%$ & $12.8 \%$ \\
\hline
\end{tabular}

Efficient energy usage is a fundamental aspect in WSN deployment. Using the maximum $P_{T x}$ results in reduced message collision and improved delivery, as a greater number of neighbours is aware of the transmission. On the downside, it will increase the total energy consumption of the network. This is more noticeable in the central nodes, as they have reduced transmission range, hence smaller coverage. It represents a trade-off between successful message delivery and the mean energy per delivered bit. To evaluate further, table $\mathrm{V}$ provides the estimated energy needed by all nodes during each period and the efficiency of each algorithm under the extreme conditions of maximum and minimum $P_{T x}$. This efficiency is measured as the amount of energy per bit delivered successfully.

TABLE V: Energy efficiency of the different algorithms, measured in Energy per transmission period and Energy/bit delivered

\begin{tabular}{ccc} 
Access method & Energy $(m J)$ & Energy/bit $(\mu J)$ \\
\hline No contention, min $P_{T x}$ & 155.0 & 211.7 \\
No contention, max $P_{T x}$ & 200.0 & 273.3 \\
Contention, min $P_{T x}$ & 152.0 & 197.5 \\
Contention, max $P_{T x}$ & 185.5 & 228.0 \\
Two transmissions, min $P_{T x}$ & 158.8 & 202.4 \\
Two transmissions, max $P_{T x}$ & 202.5 & 231.0 \\
Flat, min $P_{T x}$ & 256.7 & 325.0 \\
Flat, max $P_{T x}$ & 260.0 & 295.3 \\
1st distribution, min $P_{T x}$ & 257.3 & 348.5 \\
1st distribution, max $P_{T x}$ & 261.4 & 309.2 \\
2nd distribution, min $P_{T x}$ & 256.8 & 332.8 \\
2nd distribution, max $P_{T x}$ & 260.4 & 299.0 \\
\hline
\end{tabular}

Among the different techniques, channel listening improves energy consumption over the simple transmission proposals. It does so because it avoids unnecessary waste from collision between close nodes. Re-transmitting once increases the energy consumption, with a significant difference when using maximum power. Multiple re-transmission schemes show the greatest energy consumption between the alternatives. Moreover, the cost of delivering each bit is the lowest with the simple channel listening.

Among the most successful algorithms, the simple retransmission with maximum $P_{T x}$ represents the best effort, as the $33 \%$ increase in total energy used per period (when compared to the smallest value using simple channel listening and minimum $P_{T x}$ ). This represents $17 \%$ loss in efficiency to provide $10.4 \%$ improvement in message delivery. Moreover, the number of re-transmissions is controlled, hence reducing the probability of collisions and energy waste towards the end of $T$.

\section{CONCLUSIONS}

Routing protocols are associated with the greatest energy savings achievable by sensor nodes and ultimately the whole sensor network. Medium access is part of the network stack and therefore part of the optimisation challenge. As the issues with message delivery success are often hidden from the routing layer, it is common to ignore them when designing new protocols. Retransmissions are performed seamlessly by the lower layers and only the final report is sent to the routing layer. As result, unless the lower layers are understood 
and successfully designed in cross-layer approaches, it is not possible to achieve the best compromise.

This paper focused on the medium access problem and how it influences not only the energy consumption, but also the message delivery rate. The division in different transmission periods represents a realistic approach to environments where, due to mobility and obstacles, nodes have limited time to transmit their data successfully.

From the simulations, it becomes clear that the use of maximum $P_{T x}$ achieves the best delivery rate, compared to minimum $P_{T x}$. This difference is more relevant in nodes closer to the $\mathrm{CH}$, since they save more energy at the cost of increasing the probability of hidden node collisions. It was also found that the contention-base protocols can successfully deliver over $85 \%$ of the messages, while avoiding the excess overhead used by RTS/CTS methods and the complexity of TDMA-like schemes.

Among the different options, it is noticeable that even a simple contention mechanism without any re-transmission can be very successful while keeping the energy budged low. Moreover, the delivery rate can be increased by aggregating older data into the current message. If the packet size is unchanged, redundancy will reduce the data loss probability without increasing the transmission power. The only cost may come from further message processing, however since this is significantly smaller than the cost of extra data transmission, it can be neglected.

From the algorithms presented, two-transmission scheme shows the best compromise between message delivery and energy spent. In comparison, simple channel listening has a significantly lower message delivery, while the extra energy used by multiple retransmission schemes does not justify the small increase in successful delivery rate.

Further work will focus on dividing the MAC access in different regions and optimising the transmission power according to the node's location in relation to the cluster head.

\section{REFERENCES}

[1] P. Barbosa, N. White, and N. Harris, "Wireless sensor network for localized maritime monitoring," Advanced Information Networking and Applications - Workshops, 2008. AINAW 2008. 22nd International Conference on, pp. 681-686, March 2008.

[2] W. Ye and J. Heidemann, "Medium access control in wireless sensor networks," Wireless sensor networks, pp. 73-91, 2004

[3] W. Ye, J. Heidemann, and D. Estrin, "An energy-efficient mac protocol for wireless sensor networks," INFOCOM 2002. Twenty-First Annual Joint Conference of the IEEE Computer and Communications Societies. Proceedings. IEEE, vol. 3, pp. 1567-1576 vol.3, 2002.

[4] C. Enz, A. El-Hoiydi, J.-D. Decotignie, and V. Peiris, "Wisenet: an ultralow-power wireless sensor network solution," Computer, vol. 37, no. 8, pp. 62-70, Aug. 2004.

[5] V. Rajendran, K. Obraczka, and J. J. Garcia-Luna-Aceves, "Energyefficient collision-free medium access control for wireless sensor networks," in SenSys '03: Proceedings of the 1st international conference on Embedded networked sensor systems, 2003, pp. 181-192.

[6] G. Lu, B. Krishnamachari, and C. Raghavendra, "An adaptive energyefficient and low-latency mac for data gathering in wireless sensor networks," Parallel and Distributed Processing Symposium, 2004. Proceedings. 18th International, April 2004.

[7] S. Methley, M. Crisp, J. Newman, M. Rio, S. Bhatti, P. Ramsdale, and A. Atefi, "Efficient mobile mesh networking: attractions, myths and techno-economic roadmap to successful commercial innovation," New Frontiers in Dynamic Spectrum Access Networks, 2005. DySPAN 2005. 2005 First IEEE International Symposium on, pp. 361-370, Nov. 2005.
[8] D. Ganesan, B. Krishnamachari, A. Woo, D. Culler, D. Estrin, and S. Wicker, "An empirical study of epidemic algorithms in large scale multihop wireless networks," 2002, submitted for publication, February 2002. [Online]. Available: citeseer.ist.psu.edu/article/ganesan02empirical.html

[9] B. Yin, H. Shi, and Y. Shang, "Analysis of energy consumption in clustered wireless sensor networks," Wireless Pervasive Computing, 2007. ISWPC '07. 2nd International Symposium on, 2007.

[10] C. Chen, J. Ma, and K. Yu, "Designing energy-efficient wireless sensor networks with mobile sinks," in WSW at Sinsys'06, 2006.

[11] N. Vlajic and D. Xia, "Wireless sensor networks: To cluster or not to cluster?" in WOWMOM '06: Proceedings of the 2006 International Symposium on on World of Wireless, Mobile and Multimedia Networks, 2006, pp. 258-268.

[12] H. Yang and B. Sikdar, "Optimal cluster head selection in the leach architecture," Performance, Computing, and Communications Conference, 2007. IPCCC 2007. IEEE International Conference on, pp. 93-100, 2007.

[13] O. Arpacioglu and Z. J. Haas, "On the scalability and capacity of wireless networks with omnidirectional antennas," in IPSN '04: Proceedings of the third international symposium on Information processing in sensor networks, 2004, pp. 169-177.

[14] RFM Monolithics, Inc. [Online], RFM 802.15.4 network devices, www.rfm.com.

[15] I. Demirkol, C. Ersoy, and F. Alagoz, "Mac protocols for wireless sensor networks: a survey," Communications Magazine, IEEE, vol. 44, no. 4, pp. 115-121, April 2006.

[16] IEEE 802.15.4 WPAN-LR Task Group, "Standard for part 15.4: Wireless mac and phy specifications for low rate wpan," IEEE Computer Society, September 2006.

[17] E. W. Weisstein from MathWorld [Online], Birthday problem, http://mathworld.wolfram.com/BirthdayProblem.html.

[18] Texas Instruments, Inc. [Online], CC2430 datasheet, www.ti.com. 